\title{
Complexity, symmetry and variability of forward and backward walking at different speeds and transfer effects on forward walking: implications for neural control
}

Gregory S Walsh ${ }^{\mathrm{a}}$, Zoe Taylor

${ }^{a}$ Department of Sport, Health Sciences and Social Work, Oxford Brookes University, Oxford, UK, OX23 0BP

Corresponding Author: Dr Gregory S Walsh

gwalsh@brookes.ac.uk

$+441865483704$

Please note: This version is the authors' accepted manuscript which has been peer reviewed and accepted by the journal. Please use the DOI 10.1016/j.jbiomech.2019.109377 to find the published version of this manuscript. When citing this work use there citation:

Walsh GS and Taylor Z. (2019). Complexity, symmetry and variability of forward and backward walking at different speeds and transfer effects on forward walking: implications for neural control. Journal of Biomechanics, DOI: 10.1016/j.jbiomech.2019.109377. 


\begin{abstract}
This study aimed to investigate effects of walking direction and speed on gait complexity, symmetry and variability as indicators of neural control mechanisms, and if a period of backward walking has acute effects on forward walking. Twenty-two young adults attended 2 visits. In each visit participants walked forwards at preferred walking speed (PWS) for 3-minutes (pre) followed by 5-minutes walking each at $80 \%, 100 \%$ and $120 \%$ of PWS of either forward or backward walking then a further 3-minutes walking forward at PWS (post). The order of walking speed in each visit was randomised and walking direction of each visit was randomised. An inertial measurement unit was placed over L5 vertebra to record tri-axial accelerations. From the trunk accelerations multiscale entropy, harmonic ratio and stride time variability were calculated to measure complexity, symmetry and variability for each walk. Complexity increased with increasing walking speed for all axes in forward and backward walking, and backward walking was less complex than forward walking. Stride time variability was also greater in backward than forward walking. Anterio-posterior and medio-lateral complexity increased following forward and backward walking but there was no difference between forward and backward walking post effects. No effects were found for harmonic ratio. These results suggest during backward walking trunk motion is rigidly controlled but central pattern generators responsible for temporal gait patterns are less refined for backward walking. However, in both directions complexity increased as speed increased suggesting additional constraint of trunk motion, normally characterised by reduced complexity, is not applied as speed increases.
\end{abstract}

\title{
Keywords
}

Multiscale entropy; gait; backward walking; complexity; harmonic ratio; inertial measurement unit 


\section{Introduction}

Forward walking can be achieved through the activation of spinal networks with modulation by supraspinal activity, integrating sensory information (Merkulyeva et al., 2018) to producing a stable gait pattern that is variable within narrow bounds in healthy younger individuals. However, there is currently no consensus regarding the similarities or differences in the neural control of forward (FW) and backward (BW) walking. Previous work has suggested that FW and BW and running may utilise the same neural circuits (Duysens et al., 1996; Mehdizadeh et al., 2015). This position is supported by similarities between BW and time-reversed FW gait characteristics (Grasso et al., 1998), and the reversal of afferent sensory feedback reflex actions, consistent with a single network or central pattern for FW and BW (Duysens et al., 1996). Conversely, findings from split-belt walking indicate that the adaption of gait patterns are independent for walking direction and each leg, suggesting functional networks which can operate independently for the control of FW and BW (Choi and Bastian, 2007). Furthermore, BW has different force and electromyographic patterns to FW, rather than simply time reversed (Mahaki et al., 2017), and requires greater supraspinal activity than FW (Jansen et al., 2012; Kurz et al., 2012), however, this is not a consistent finding (Groff et al., 2019).

BW has regularly demonstrated greater variability than FW, with higher variability reported for stride time (Bollens et al., 2014; Choi and Bastian, 2007; Kurz et al., 2012), muscle activations (Ivanenko et al., 2008) and joint angles (Kastavelis et al., 2010), and joint angle coordination in backward running (Mehdizadeh et al., 2015). Greater variability is also associated with increased activity of the supplementary motor area and pre-central gyrus in humans (Kurz et al., 2012) and cats (Zelenin et al., 2011). The increased cortical activity suggests greater neural resources are responsible for producing the BW pattern, possibly reducing the available resources to maintain the stability and consistency of the gait pattern or respond to perturbations and instability (Kurz et al., 2012).

A significant body of work has focussed on determining the control dynamics of FW using a variety of linear and non-linear measures (Bruijn et al., 2009; Costa et al., 2003; Dierick et al., 2017; Dingwell and Cusumano, 2010). For example, in FW the stride interval exhibits persistent long-range 
autocorrelations, i.e. Hurst and $\alpha$-exponents, (Dierick et al., 2017; Dingwell and Cusumano, 2010), increasing walking speed reduces local dynamic stability, i.e. largest Lyapunov exponent, (Bruijn et al., 2009) but increases symmetry, i.e. harmonic ratio, (Huijben et al., 2018), and complexity of trunk motion, i.e. multiscale entropy (MSE), changes across the lifespan with the development of the gait pattern (Bisi and Stagni, 2016). Recent studies have demonstrated that BW has similar long-range autocorrelations of stride intervals compared to FW (Bollens et al., 2014), or greater long-range autocorrelations suggesting the stride interval is more predictable in BW than FW (Dierick et al., 2017), and that increasing speed decreases local dynamic stability of trunk motion of backwards running (Mehdizadeh et al., 2014). Non-linear analyses provide insight into the neural control dynamics of locomotion (Bruijn et al., 2009; Costa et al., 2003; Dierick et al., 2017; Dingwell and Cusumano, 2010). For example, the difference in MSE of the stride time interval in self-paced walking compared to shuffled surrogate data and the similarity of MSE for metronomically paced walking, where supra-spinal pacesetters are constrained, compared to surrogate data has demonstrated that the control of the stride interval is dependent on supra-spinal control (Costa et al., 2003). However, there is currently limited understanding of these non-linear control dynamics in BW and the effects of added task constraints such as changing walking speed.

It has recently been demonstrated that periods of 6 minutes treadmill FW or BW have differing acute effects on standing posture with increased forward inclination after FW and backward inclination and centre of pressure position after BW (De Nunzio et al., 2009). Adaptions in FW following BW may demonstrate the adaptability or flexibility of the control systems and it can be suggested that if the two locomotion patterns share the same neural control structures that BW will cause changes in subsequent FW (Choi and Bastian, 2007). However, during split belt walking BW did not cause acute adaptations in FW as measured by coordination of leg kinematics (Choi and Bastian, 2007). This indicates the of resistance to change central pattern generators responsible for limb motion in each direction in response to the different neuromuscular task. Analysis of gait complexity could better identify transfer effects on control of gait dynamics that may arise from the more novel (in comparison to FW) BW task. Understanding the acute effects of BW on FW has been studied as it has 
become increasingly popular to include BW interventions for injury rehabilitation and in clinical populations (e.g. Hoogkamer et al., 2014; Rose et al., 2018) and therefore a greater understanding of any acute effects can both inform future interventions and possible responses to these interventions.

The aim of this study was to determine the effects of walking direction and speed on gait complexity, symmetry and stride time variability as indicators of neural control mechanisms, and if a period of BW has an acute effect on FW. It was hypothesised that (1) BW would be more complex, less smooth and more variable than $\mathrm{FW}$ in response to the greater task demand of $\mathrm{BW},(2)$ that increasing speed would increase complexity and stride time variability and reduce symmetry with greater effects in BW than FW as neural resources are dedicated maintaining a speed greater than preferred, and (3) that BW would not result in post effects in FW due to the resistance of FW control mechanisms to interference by the BW task.

\section{Methods}

\section{Participants}

Twenty-two participants volunteered for this study (n-male: 11, n-female: 11, age: $21 \pm 1$ years, height: $1.79 \pm 0.10 \mathrm{~m}$, mass: $77.8 \pm 11.1 \mathrm{~kg}$ ). Participants were free of lower limb injury and neurological conditions. All participants were informed of the nature of the study and provided written informed consent before completing any study procedures. All protocols were conducted in compliance with the declaration of Helsinki. The study received ethical approval from the Oxford Brookes University Research Ethics Committee.

\section{Procedures}

All participants attended two laboratory visits. In each visit participants complete 6 minutes of FW or BW treadmill walking familiarisation (Meyer et al., 2019). To determine preferred walking speed (PWS) participants were allowed to manually increase and decrease treadmill speed until they reached their perceived preferred walking speed. 
Treadmill familiarisation was followed by 3 minutes of FW at the forward PWS (Pre). Participants then completed 5 minutes walking at 80\% (PWS80), 100\% (PWS100) and 120\% (PWS120) PWS, 15 minutes total, of either FW or BW with no break between speeds. The 15 minutes FW or BW was followed immediately by a further 3 minutes of FW at PWS (Post). In the second visit participants completed the same protocol but performed walking in the opposite direction for the PWS80, PWS100 and PWS120 trials than was performed in the first visit. Treadmill speed was adjusted while participants walked on the treadmill and each 5 minute period was started once the treadmill had reached the required speed. The visit order (forward or backward walking) and walking speed trial order were randomised.

At the start of each visit before the Pre FW condition participants were fitted with a 9-axis inertial measurement unit (IMU: LPMS-B2, Life Performance Research, Tokyo, Japan) over the L5 vertebra such that the positive acceleration sensing axes were in the upward, right and forward directions respectively. The IMU recorded 3-axis acceleration, 3-axis gyroscope, and 3-axis magnetometer data at $100 \mathrm{~Hz}$.

\section{Data analysis}

\section{Data processing}

For each Pre, Post, PWS80, PWS100 and PWS120 trial, the middle 15000 data samples were used for analysis. The acceleration signals were not filtered to ensure all relevant information was retained (Bisi and Stagni, 2016; Riva et al., 2013). All data analysis was completed using custom MATLAB programmes (2016b, The MathWorks, Inc., Natick, USA).

\section{Multiscale entropy}

Multiscale entropy (e.g. Bisi and Stagni, 2016; Borg and Laxåback, 2010; Costa et al., 2005, 2003) was used to determine complexity of FW and BW separately for the AP, ML and VT axes accelerations. Consecutively more course-grained time series were calculated from the original acceleration signals by averaging data points in non-overlapping windows of length $\tau, \tau$ ranged from 
1-6 data points. From each course-grained time series the sample entropy (SE) was calculated as the negative natural logarithm of the conditional probability, $\mathrm{C}(r)$, that two sequences of $m$ consecutive data points which are within a radius of $r \delta$ of each other, where $\delta$ is the standard deviation of the original signal, will remain close when one more point is included:

$$
S E=-\ln \frac{C^{m+1}(r)}{C^{m}(r)}
$$

The value of $m$ and $r$ were set at 2 and 0.2 respectively (Bisi et al., 2018; Bisi and Stagni, 2016; Riva et al., 2013).

After calculation of the sample entropy at each course-grained time scale, the complexity index (CI) was calculated as the area under the curve of SE vs. $\tau$ to provide an indication of overall complexity:

$$
C I=\sum_{i=1}^{6} S E(i)
$$

A higher CI value represents greater complexity.

\section{Harmonic ratio}

Harmonic ratio (HR) was calculated from the Fast Fourier Transforms of the AP, ML and VT accelerations to determine the symmetry of gait. For the AP and VT axes the summed amplitudes of the first 10 even harmonics were divided by the summed amplitudes of the first 10 odd harmonics and for the ML axis HR was calculated as the summed amplitudes of the first 10 odd harmonics divided by the summed amplitudes of the first 10 even harmonics (Riva et al., 2013). The difference in calculation of HR for the different axes is due to the fact that the AP and VT signals have two periods per stride, therefore the second and subsequent even harmonics are dominant in the signal, whereas, 
the ML signal has one period per stride, therefore the first and subsequent odd harmonics are dominant in the signal. A higher HR is considered to represent more symmetric gait.

\section{Stride time variability}

To determine the stride time variability $\left(\mathrm{ST}_{\mathrm{VAR}}\right)$ in each $\mathrm{FW}$ and $\mathrm{BW}$ walking trial heel-strike events were detected using a method described previously (McCamley et al., 2012; Pacini Panebianco et al., 2018; Zijlstra and Hof, 2003). Briefly, heel-strikes were detected as the peaks of the filtered $\left(4^{\text {th }}\right.$ order, dual-pass Butterworth filter with a $2 \mathrm{~Hz}$ cut-off frequency) AP acceleration signal occurring immediately before the zero-crossing from positive to negative. For BW walking trials the same protocol was applied to the inverse of the AP acceleration signal. Stride time was then determined as the time between every second heel-strike, i.e. 2 steps per stride, and the $\mathrm{ST}_{\mathrm{VAR}}$ was the standard deviation of the calculated stride times for each trial.

\section{Statistics}

All data were tested for normality using the Shapiro-Wilk test. To test the effect of the FW and BW protocols on PWS FW before (Pre) and after (Post) 15 minutes walking on CI, HR and ST VAR a 2 (Pre and Post) x 2 (FW and BW) two-way repeated measures MANOVA was performed. To determine the effect of walking speed and direction on CI, HR and ST VAR $_{3} 3$ (PWS80, PWS100 and PWS120) x 2 (FW and BW) repeated measures MANOVA was performed. The Wilk's Lambda test statistic was used for all MANOVA.

For all significant multivariate main and interaction effects univariate two-way ANOVA were performed for each variable to determine where significant differences were present. For significant univariate effects of walking speed post hoc pairwise comparisons with a Bonferroni correction were performed. If main effects of walking speed violated the assumption of sphericity the GreenhouseGeisser correction of the degrees of freedom was performed. For all MANOVA and ANOVA main effects the partial eta squared $\left(\eta_{p}^{2}\right)$ effect size was calculated, values of $0.01,0.06$ and 0.14 represent 
small, moderate and large effect sizes respectively. For all tests the alpha level of significance was set at $\mathrm{p}<0.05$. All statistical analysis was performed using SPSS (version 25, IBM Corp., NY USA).

\section{Results}

\section{Pre and post comparisons}

Multivariate effects

There was a significant multivariate effect of time $\left(\lambda=0.38, \mathrm{~F}_{7,13}=3.10, \mathrm{p}=0.037, \eta_{p}^{2}=0.63\right)$ on $\mathrm{CI}, \mathrm{HR}$ and ST VAR during Pre and Post PWS FW, however, there were no effects for walking direction $\left(\lambda=0.74, \mathrm{~F}_{7,13}=0.67, \mathrm{p}=0.698, \eta_{p}^{2}=0.26\right)$ or interaction effects $\left(\lambda=0.60, \mathrm{~F}_{7,13}=1.23, \mathrm{p}=0.356, \eta_{p}^{2}=0.40\right)$ on CI, HR and $\mathrm{ST}_{\mathrm{VAR}}$. The mean and standard deviation of all variables pre and post walking are shown in Table 1.

Univariate effects of time

There were significant effects of time for $\mathrm{CI}$ in the AP $\left(\mathrm{F}_{1,19}=18.33, \mathrm{p}<0.001\right)$ and $\mathrm{ML}\left(\mathrm{F}_{1,19}=4.64\right.$, $\mathrm{p}=0.044)$ axes, AP and ML CI were both greater post than pre. However, there were no other significant univariate effects for $\mathrm{CI}, \mathrm{HR}$ or $\mathrm{ST}_{\mathrm{VAR}}$.

Table 1. Mean, standard deviation and univariate main effect partial eta squared $\left(\eta_{p}^{2}\right)$ effect size of the complexity index (CI) and harmonic ratio (HR) in the anterio-posterior (AP), medio-lateral (ML) and vertical (VT) axes and stride time variability ( $\mathrm{ST}_{\mathrm{VAR}}$ ) during preferred walking speed forward walking before (Pre) and after (Post) 15 minutes forward (FW) or backward (BW) walking.

\begin{tabular}{|c|c|c|c|c|c|c|c|c|}
\hline & & FW & & BW & & $\eta_{p}^{2}$ & $\eta_{p}^{2}$ & $\eta_{p}^{2}$ \\
\hline & & Pre & Post & Pre & Post & Direction & Time & Interaction \\
\hline CI & AP & $0.98 \pm 0.30$ & $1.00 \pm 0.28$ & $0.94 \pm 0.30$ & $1.01 \pm 0.28$ & 0.01 & $0.49 *$ & 0.14 \\
\hline & ML & $1.17 \pm 0.58$ & $1.25 \pm 0.60$ & $1.10 \pm 0.52$ & $1.17 \pm 0.52$ & 0.08 & $0.20 *$ & $<0.01$ \\
\hline & VT & $1.75 \pm 0.64$ & $1.68 \pm 0.68$ & $1.68 \pm 0.65$ & $1.74 \pm 0.64$ & $<0.01$ & $<0.01$ & 0.13 \\
\hline HR & AP & $3.12 \pm 0.24$ & $3.05 \pm 0.31$ & $3.21 \pm 0.34$ & $3.12 \pm 0.22$ & 0.07 & 0.06 & $<0.01$ \\
\hline
\end{tabular}




\begin{tabular}{lcccccccc}
\hline & ML & $3.00 \pm 0.17$ & $3.01 \pm 0.23$ & $3.00 \pm 0.22$ & $2.95 \pm 0.23$ & 0.07 & $<0.01$ & $<0.01$ \\
& & & & & & & & \\
& & $3.15 \pm 0.24$ & $3.03 \pm 0.22$ & $3.24 \pm 0.34$ & $3.12 \pm 0.19$ & 0.09 & 0.15 & $<0.01$ \\
$\mathrm{ST}_{\text {VAR }}$ (s) & & $0.02 \pm 0.01$ & $0.03 \pm 0.01$ & $0.03 \pm 0.04$ & $0.03 \pm 0.02$ & 0.13 & 0.01 & 0.03 \\
\hline
\end{tabular}

* indicates a main effect of time, Post is greater than Pre

\section{Effects of walking speed and direction}

Multivariate effects

There were significant multivariate effects of walking speed $\left(\lambda=0.01, \mathrm{~F}_{14,8}=61.19, \mathrm{p}<0.001, \eta_{p}^{2}=0.99\right)$ and walking direction $\left(\lambda=0.16, \mathrm{~F}_{7,15}=11.61, \mathrm{p}<0.001, \eta_{p}^{2}=0.84\right)$ on $\mathrm{CI}, \mathrm{HR}$ and $\mathrm{ST}_{\mathrm{VAR}}$, however, there was no interaction effect $\left(\lambda=0.34, \mathrm{~F}_{14,8}=1.11, \mathrm{p}=0.458, \eta_{p}^{2}=0.66\right)$. The mean and standard deviation of all variables for each speed are shown in Table 2.

Univariate effects of walking speed

There were univariate effects of speed on $\mathrm{CI}$ in the $\mathrm{AP}\left(\mathrm{F}_{2,42}=106.22, \mathrm{p}<0.001\right), \mathrm{ML}\left(\mathrm{F}_{1.2,25.9}=92.15\right.$, $\mathrm{p}<0.001)$ and VT $\left(\mathrm{F}_{2,42}=128.48, \mathrm{p}<0.001\right)$ axes. For all axes, CI in PWS100 and PWS120 were greater than PWS80 (all $\mathrm{p}<0.001$ ) and greater in PWS120 than PWS100 (all $\mathrm{p}<0.001$ ). No speed effects were found for HR in the AP, ML or VT axes, or for $\mathrm{ST}_{\mathrm{VAR}}$.

Univariate effects of walking direction

There were univariate effects of walking direction on $\mathrm{CI}$ in the AP $\left(\mathrm{F}_{1,21}=11.46, \mathrm{p}=0.003\right)$, ML (F$\left.{ }_{1,21}=25.46, \mathrm{p}<0.001\right)$ and VT $\left(\mathrm{F}_{1,21}=56.79, \mathrm{p}<0.001\right)$ axes, and on $\mathrm{ST}_{\mathrm{VAR}}\left(\mathrm{F}_{1,21}=5.77, \mathrm{p}=0.026\right)$. The $\mathrm{CI}$ was greater for all axes in $\mathrm{FW}$ than $\mathrm{BW}$ and $\mathrm{ST}_{\mathrm{VAR}}$ was greater in $\mathrm{BW}$ than $\mathrm{FW}$. However, there was no effect of walking direction on HR for the AP, ML or VT axes.

Table 2. Mean, standard deviation and univariate main effect partial eta squared $\left(\eta_{p}^{2}\right)$ effect size of the complexity index $(\mathrm{CI})$ and harmonic ratio (HR) for the anterio-posterior (AP), medio-lateral (ML) and vertical (VT) axes and stride time variability $\left(\mathrm{ST}_{\mathrm{VAR}}\right)$ during forward $(\mathrm{FW})$ and backward $(\mathrm{BW})$ walking at $80 \%$ (PWS80), 100\% (PWS100) and 120\% (PWS120) of preferred walking speed. 


\begin{tabular}{|c|c|c|c|c|c|c|c|c|c|c|}
\hline & & FW & & & BW & & & $\eta_{p}^{2}$ & $\eta_{p}^{2}$ & $\eta_{p}^{2}$ \\
\hline & & PWS80 & PWS100 & PWS120 & PWS80 & PWS100 & PWS120 & Direction & Speed & Interaction \\
\hline \multirow[t]{3}{*}{$\mathrm{CI}$} & AP & $0.78 \pm 0.25$ & $1.01 \pm 0.27 *$ & $1.19 \pm 0.28 * \dagger$ & $0.65 \pm 0.27$ & $0.76 \pm 0.32 *$ & $1.01 \pm 0.31 * \dagger$ & $0.35^{\ddagger}$ & $0.84^{\mp}$ & 0.12 \\
\hline & ML & $0.83 \pm 0.45$ & $1.23 \pm 0.61 *$ & $1.71 \pm 0.82 * \dagger$ & $0.42 \pm 0.27$ & $0.61 \pm 0.36^{*}$ & $0.97 \pm 0.51 * \dagger$ & $0.55^{\ddagger}$ & $0.81^{*}$ & 0.18 \\
\hline & $\mathrm{VT}$ & $1.26 \pm 0.53$ & $1.73 \pm 0.66^{*}$ & $2.03 \pm 0.64 * \dagger$ & $0.54 \pm 0.36$ & $0.80 \pm 0.43 *$ & $1.23 \pm 0.59 * \dagger$ & $0.73^{\ddagger}$ & $0.86^{¥}$ & 0.09 \\
\hline \multirow[t]{3}{*}{ HR } & AP & $2.95 \pm 0.28$ & $3.09 \pm 0.30$ & $3.10 \pm 0.28$ & $3.12 \pm 0.24$ & $3.06 \pm 0.30$ & $3.10 \pm 0.27$ & 0.04 & 0.03 & 0.08 \\
\hline & ML & $2.95 \pm 0.19$ & $3.15 \pm 0.39$ & $2.99 \pm 0.24$ & $2.97 \pm 0.21$ & $2.96 \pm 0.24$ & $2.94 \pm 0.21$ & 0.07 & 0.08 & 0.09 \\
\hline & VT & $2.97 \pm 0.26$ & $3.05 \pm 0.24$ & $3.06 \pm 0.24$ & $3.09 \pm 0.23$ & $3.08 \pm 0.31$ & $3.12 \pm 0.38$ & 0.12 & 0.03 & 0.01 \\
\hline $\mathrm{ST}_{\mathrm{VAR}}$ & & $0.04 \pm 0.02$ & $0.02 \pm 0.01$ & $0.02 \pm 0.01$ & $0.08 \pm 0.04$ & $0.14 \pm 0.36$ & $0.05 \pm 0.02$ & $0.22^{\#}$ & 0.05 & 0.05 \\
\hline
\end{tabular}

(s)

\footnotetext{
* indicates that value is greater than PWS80

$\dagger$ indicates that PWS120 is greater than PWS100

$¥$ indicates a main effect of direction, $\mathrm{FW}$ is greater than BW

\# indicates a main effect of direction, BW is greater than FW

$¥$ indicates a main effect of speed
}

\section{Discussion}

This study aimed to determine the effects of walking direction and speed on the complexity, symmetry and stride time variability of gait, and to determine if post effects of a period of forward and backward walking were present in FW. The results demonstrated that FW is more complex, with lower ST VAR than BW and that in both walking directions increasing walking speed increases the complexity of gait. AP and ML complexity were also greater Post walking than Pre, however there was no interaction with walking direction.

The lower complexity of BW than FW in the present study could be indicative of the central nervous system exerting additional constraint of the motion of the centre of mass (Borg and Laxåback, 2010), in support of findings of greater supplementary motor area and pre-central gyrus activity in BW than 
FW in human (Kurz et al., 2012) and animal models (Zelenin et al., 2011). This interpretation is congruous with previous work demonstrating that the stride interval of BW is less complex and more predictable than FW (Dierick et al., 2017). During FW visual feedback is used to control speed, stability and accurate foot placement (Reynolds and Day, 2005), however in BW the visual information is removed which may require a reweighting of sensory information, increasing demand on neural resources to control BW (Kurz et al., 2012). The lower complexity of BW could also be due to the different anatomical and mechanical constraints of BW, for example the reduced knee and hip range of motion (Kastavelis et al., 2010).

The present study also demonstrated that $\mathrm{ST}_{\mathrm{VAR}}$ was greater in $\mathrm{BW}$ than $\mathrm{FW}$ which is in agreement with previous findings (Bollens et al., 2014; Choi and Bastian, 2007; Kurz et al., 2012). When considered together, the greater $\mathrm{ST}_{\mathrm{VAR}}$ and lower complexity in $\mathrm{BW}$ than $\mathrm{FW}$, it could be hypothesised that when BW, stability of trunk motion is actively controlled to a greater extent than the spatio-temporal gait pattern. It is possible that the temporal structure of the gait pattern is generated by a central pattern (Lacquaniti et al., 2012) with minimal supraspinal input allowing greater fluctuation in stride time interval, with additional neural resources assigned for controlling trunk motion. It could also be suggested that the greater $\mathrm{ST}_{\mathrm{VAR}}$ in $\mathrm{BW}$ is the result of a separate, less refined neural control network for BW compared to FW (Kurz et al., 2012). However, in contrast, the robustness of gait symmetry, as indicated by no change in HR, to changes in walking speed and direction could indicate that FW and BW have similar control networks which produce harmonics that are intrinsic to the walking pattern irrespective of walking direction or speed (Pasciuto et al., 2017) in agreement with the notion that structure of the gait pattern is produced by similar central pattern generators.

Complexity increased with increasing walking speed but with no interaction effect for walking direction in the present study. This finding may indicate that although the control required for BW is different to FW, both have a similar response to increased speed. Previous findings in backward and forward running have found that dynamic stability decreases with increasing speed similarly in both 
directions (Mehdizadeh et al., 2014). During FW, activity of the prefrontal cortex is increased with increasing walking speed (Hamacher et al., 2015; Harada et al., 2009; Suzuki et al., 2004). The prefrontal cortex is, in part, responsible for intentional movement control (Harada et al., 2009; Lau et al., 2004), which may be required for producing non-preferred walking speeds, reducing available neural resources for the control of gait stability and complexity. Increased movement complexity can be indicative of decreased constraint on the system (Cavanaugh et al., 2007). The increase in complexity with increasing walking speed could indicate that with sufficient redundancy in the control system, the central nervous system allows the complexity to increase without additional constraint, thereby, reducing neural demands, however direct evidence for this interpretation is beyond the scope of the results of the present study and should be considered for future research.

This study found no difference in the effect of 15 minutes BW and FW on post FW at PWS, however, AP and ML complexity was greater post than pre for both directions. The current findings are in agreement with previous results that found no adaptation in FW kinematic coordination following split belt $\mathrm{BW}$, suggesting this indicates that $\mathrm{FW}$ and $\mathrm{BW}$ are controlled by functionally separate networks (Choi and Bastian, 2007). The cause for the increase in complexity following both FW and BW is not immediately clear but could be the result of further adaptation to the treadmill walking or fatigue effects (Cortes et al., 2014).

This study has limitations which must be considered. The study was restricted to healthy young adults so extension of these findings to clinical populations or different age groups should be avoided. Furthermore, walking speed was not controlled for between conditions. It was chosen to use direction specific PWS, and increments of PWS, in order to examine the participants' central nervous systems preferred behaviour in each direction and the effect of perturbation away from this preferred state. Finally, the study of gait in this experiment was limited to treadmill walking which produces a different gait pattern when compared to overground walking and therefore comparisons to findings of studies reporting overground walking only must be made with caution, however, treadmill offers the 
opportunity for controlled and constant speed conditions and easy collection of a large number of continuous strides.

\section{Conclusion}

This study has demonstrated that BW is less complex than FW but has greater $\mathrm{ST}_{\mathrm{VAR}}$ indicating that the trunk motion is tightly controlled during $\mathrm{BW}$, or is constrained by the mechanics of $\mathrm{BW}$, but that the central pattern generator responsible for temporal gait patterns in BW is possibly less refined than that of FW. It was also demonstrated that in both FW and BW increasing speed increases complexity in the absence of changes in $\mathrm{ST}_{\mathrm{VAR}}$ and $\mathrm{HR}$ which could suggest redundancy in the control networks for trunk motion of FW and BW allowing complexity to increase without the need for additional constraint. Finally, there was no difference in the effect of FW and BW on FW at PWS, in agreement with previous findings, which may be indicative of separate control networks for each walking direction. However, both walking directions increased complexity in post walking.

\section{Conflict of interest statement}

The authors have no conflicts of interest to declare. This study received no external funding.

\section{References}

Bisi, M.C., Stagni, R., 2016. Complexity of human gait pattern at different ages assessed using multiscale entropy: from development to decline. Gait Posture 47, 37-42. doi:10.1016/j.gaitpost.2016.04.001

Bisi, M.C., Tamburini, P., Panebianco, G.P., Stagni, R., 2018. Nonlinear analysis of human movement dynamics offers new insights in the development of motor control during childhood. J. Biomech. Eng. 140, 111002. doi:10.1115/1.4040939

Bollens, B., Crevecoeur, F., Detrembleur, C., Warlop, T., Lejeune, T.M., 2014. Variability of human gait: Effect of backward walking and dual-tasking on the presence of long-range autocorrelations. Ann. Biomed. Eng. 42, 742-750. doi:10.1007/s10439-013-0961-9

Borg, F.G., Laxåback, G., 2010. Entropy of balance - some recent results. J. Neuroeng. Rehabil. 7, 38. doi:10.1186/1743-0003-7-38 
Bruijn, S.M., van Dieën, J.H., Meijer, O.G., Beek, P.J., 2009. Is slow walking more stable? J.

Biomech. 42, 1506-1512. doi:10.1016/j.jbiomech.2009.03.047

Cavanaugh, J.T., Mercer, V.S., Stergiou, N., 2007. Approximate entropy detects the effect of a secondary cognitive task on postural control in healthy young adults: A methodological report. J. Neuroeng. Rehabil. 4, 1-7. doi:10.1186/1743-0003-4-42

Choi, J.T., Bastian, A.J., 2007. Adaptation reveals independent control networks for human walking. Nat. Neurosci. 10, 1055-1062. doi:10.1038/nn1930

Cortes, N., Onate, J., Morrison, S., 2014. Differential effects of fatigue on movement variability. Gait Posture 39, 888-893. doi:10.1016/j.gaitpost.2013.11.020

Costa, M., Goldberger, A.L., Peng, C.K., 2005. Multiscale entropy analysis of biological signals. Phys. Rev. E - Stat. Nonlinear, Soft Matter Phys. 71, 1-18. doi:10.1103/PhysRevE.71.021906

Costa, M., Peng, C.K., Goldberger, A.L., Hausdorff, J.M., 2003. Multiscale entropy analysis of human gait dynamics. Phys. A Stat. Mech. its Appl. 330, 53-60. doi:10.1016/j.physa.2003.08.022

De Nunzio, A.M., Zanetti, C., Schieppati, M., 2009. Post-effect of forward and backward locomotion on body orientation in space during quiet stance. Eur. J. Appl. Physiol. 105, 297-307. doi:10.1007/s00421-008-0903-7

Dierick, F., Nivard, A.L., White, O., Buisseret, F., 2017. Fractal analyses reveal independent complexity and predictability of gait. PLoS One 12, 1-18. doi:10.1371/journal.pone.0188711

Dingwell, J.B., Cusumano, J.P., 2010. Re-interpreting detrended fluctuation analyses of stride-tostride variability in human walking. Gait Posture 32, 348-353.

doi:10.1016/j.gaitpost.2010.06.004

Duysens, J., Tax, A.A.M., Murrer, L., Dietz, V., 1996. Backward and forward walking use different patterns of phase-dependent modulation of cutaneous reflexes in humans. J. Neurophysiol. 76, 301-310.

Grasso, R., Bianchi, L., Lacquaniti, F., Ogihara, N., Oku, T., Andrada, E., Blickhan, R., Nyakatura, J.A., Fischer, M.S., 1998. Motor patterns for human gait : backward versus forward locomotion. J. Neurophysiol. 80, 1868-1885. 
Groff, B.R., Antonellis, P., Schmid, K.K., Knarr, B.A., Stergiou, N., 2019. Stride-time variability is related to sensorimotor cortical activation during forward and backward walking. Neurosci. Lett. 692, 150-158. doi:10.1016/j.neulet.2018.10.022

Hamacher, Dennis, Herold, F., Wiegel, P., Hamacher, Daniel, Schega, L., 2015. Brain activity during walking: A systematic review. Neurosci. Biobehav. Rev. 57, 310-327. doi:10.1016/j.neubiorev.2015.08.002

Harada, T., Miyai, I., Suzuki, M., Kubota, K., 2009. Gait capacity affects cortical activation patterns related to speed control in the elderly. Exp. Brain Res. 193, 445-454. doi:10.1007/s00221-008$1643-\mathrm{y}$

Hoogkamer, W., Meyns, P., Duysens, J., 2014. Steps forward in understanding backward gait: From basic circuits to rehabilitation. Exerc. Sport Sci. Rev. 42, 23-29. doi:10.1249/JES.0000000000000000

Huijben, B., van Schooten, K., van Dieën, J., Pijnappels, M., Schooten, van K., Dieën, van J., 2018. The effect of walking speed on quality of gait in older adults. Gait Posture 65, 112-116. doi:10.1016/j.gaitpost.2018.07.004

Ivanenko, Y.P., Cappellini, G., Poppele, R.E., Lacquaniti, F., 2008. Spatiotemporal organization of $\alpha$ motoneuron activity in the human spinal cord during different gaits and gait transitions. Eur. J. Neurosci. 27, 3351-3368. doi:10.1111/j.1460-9568.2008.06289.x

Jansen, K., De Groote, F., Massaad, F., Meyns, P., Duysens, J., Jonkers, I., 2012. Similar muscles contribute to horizontal and vertical acceleration of center of mass in forward and backward walking: implications for neural control. J. Neurophysiol. 107, 3385-3396. doi:10.1152/jn.01156.2011

Kastavelis, D., Mukherjee, M., Decker, L., Stergiou, N., 2010. Variability of lower extremity joint kinematics during backward walking in a virtual environment. Nonlinear Dynamics. Psychol. Life Sci. 14, 165-78.

Kurz, M.J., Wilson, T.W., Arpin, D.J., 2012. Stride-time variability and sensorimotor cortical activation during walking. Neuroimage 59, 1602-1607. doi:10.1016/j.neuroimage.2011.08.084 Lacquaniti, F., Ivanenko, Y.P., Zago, M., 2012. Patterned control of human locomotion. J. Physiol. 
590, 2189-2199. doi:10.1113/jphysiol.2011.215137

Lau, H.C., Rogers, R.D., Haggard, P., Passingham, R.E., 2004. Attention to Intention. Science (80-. ). 303, 1208-1210. doi:10.1126/science.1090973

Mahaki, M., De Sá E Souza, G.S., Mimar, R., Vieira, M.F., 2017. The comparison of ground reaction forces and lower limb muscles correlation and activation time delay between forward and backward walking. Gait Posture 58, 380-385. doi:10.1016/j.gaitpost.2017.08.039

McCamley, J., Donati, M., Grimpampi, E., Mazzà, C., 2012. An enhanced estimate of initial contact and final contact instants of time using lower trunk inertial sensor data. Gait Posture 36, 316318. doi:10.1016/j.gaitpost.2012.02.019

Mehdizadeh, S., Arshi, A.R., Davids, K., 2015. Quantifying coordination and coordination variability in backward versus forward running: Implications for control of motion. Gait Posture 42, 172 177. doi:10.1016/j.gaitpost.2015.05.006

Mehdizadeh, S., Arshi, A.R., Davids, K., 2014. Effect of speed on local dynamic stability of locomotion under different task constraints in running. Eur. J. Sport Sci. 14, 791-798. doi:10.1080/17461391.2014.905986

Merkulyeva, N., Veshchitskii, A., Gorsky, O., Pavlova, N., Zelenin, P. V, Gerasimenko, Y., Deliagina, T.G., Musienko, P., 2018. Distribution of spinal neuronal networks controlling forward and backward locomotion. J. Neurosci. 38, 4695-4707. doi:10.1523/JNEUROSCI.2951-17.2018

Meyer, C., Killeen, T., Easthope, C.S., Curt, A., Bolliger, M., Linnebank, M., Zörner, B., Filli, L., 2019. Familiarization with treadmill walking: How much is enough? Sci. Rep. 9, 5232. doi:10.1038/s41598-019-41721-0

Pacini Panebianco, G., Bisi, M.C., Stagni, R., Fantozzi, S., 2018. Analysis of the performance of 17 algorithms from a systematic review: Influence of sensor position, analysed variable and computational approach in gait timing estimation from IMU measurements. Gait Posture 66, 7682. doi:10.1016/j.gaitpost.2018.08.025

Pasciuto, I., Bergamini, E., Iosa, M., Vannozzi, G., Cappozzo, A., 2017. Overcoming the limitations of the Harmonic Ratio for the reliable assessment of gait symmetry. J. Biomech. 53, 84-89. 
doi:10.1016/j.jbiomech.2017.01.005

Reynolds, R.F., Day, B.L., 2005. Visual guidance of the human foot during a step. J. Physiol. 569, 677-684. doi:10.1113/jphysiol.2005.095869

Riva, F., Toebes, M.J.P., Pijnappels, M., Stagni, R., van Dieën, J.H., 2013. Estimating fall risk with inertial sensors using gait stability measures that do not require step detection. Gait Posture 38, 170-4. doi:10.1016/j.gaitpost.2013.05.002

Rose, D.K., DeMark, L., Fox, E.J., Clark, D.J., Wludyka, P., 2018. A backward walking training program to improve balance and mobility in acute stroke: A pilot randomized controlled trial. J. Neurol. Phys. Ther. 42.

Suzuki, M., Miyai, I., Ono, T., Oda, I., Konishi, I., Kochiyama, T., Kubota, K., 2004. Prefrontal and premotor cortices are involved in adapting walking and running speed on the treadmill: An optical imaging study. Neuroimage 23, 1020-1026. doi:10.1016/j.neuroimage.2004.07.002

Zelenin, P. V, Deliagina, T.G., Orlovsky, G.N., Karayannidou, A., Stout, E.E., Sirota, M.G., Beloozerova, I.N., 2011. Activity of motor cortex neurons during backward locomotion. J. Neurophysiol. 105, 2698-2714. doi:10.1152/jn.00120.2011

Zijlstra, W., Hof, A.L., 2003. Assessment of spatio-temporal gait parameters from trunk accelerations during human walking. Gait Posture 18, 1-10. doi:10.1016/S0966-6362(02)00190-X 